\title{
Prediction of Channel Quality after Handover for Mobility Management in 5G
}

\author{
Zdenek Becvar, Pavel Mach \\ Dept. of Telecommunication Engineering, FEE \\ Czech Technical University in Prague \\ Technicka 2, 16627 Prague, Czech Republic \\ zdenek.becvar@fel.cvut.cz, machp2@fel.cvut.cz
}

\author{
Emilio Calvanese Strinati \\ CEA-Leti \\ 17 rue des Martyrs, 38000 Grenoble, France \\ emilio.calvanese-strinati@cea.fr
}

\begin{abstract}
The fifth generation of wireless networks should enable the same experience to users at home, in the office or on the move thanks to seamless handover. Call admission control (CAC) provides the means to avoid call drops due to lack of resources at a target cell during handover. The purpose of the $\mathrm{CAC}$ is to decide if handover should be initiated or if a new call can be established. A specific quantity of resources is reserved to the users entering the cell in the future to avoid call drops. A prediction of user's movement and amount of resources required by the users after handover can be performed in order to optimize amount of reserved resources. In this paper, we address prediction of the number of resources required by the users at the target cell after handover. To that end, we propose new approach for prediction of channel quality indicator (CQI) after handover. The prediction exploits knowledge of handover hysteresis and decomposition of interference into two parts. As the results show, the proposed algorithm increases ratio of successfully predicted CQI up to 1.9 times with respect to existing approaches.
\end{abstract}

Keywords- 5G, call admission control, handover, interference, prediction, mobility

\section{INTRODUCTION}

Mobility of users essentially influences radio resource management in OFDMA-based wireless networks. The reason is that the amount of physical resources allocated to a user equipment (UE) is varying with user's movement. This happens due to changing signal to interference plus noise ratio (SINR). In addition, mobility must be handled also from a handover procedure point of view. If the UE is moving, handover has to be performed to avoid a connection drop and to guarantee required QoS (Quality of Service). In the most common case, handover is performed if a target cell provides a higher quality of signal than a serving cell. With expected increase in density of small cells in $5 \mathrm{G}$ networks comparing to today $3 \mathrm{G} / 4 \mathrm{G}$ networks [1], handover becomes even more frequent, especially in densely populated areas. Besides fulfilling conditions on handover from the signal quality point of view, the target cell must be also able to offer enough radio resources to guarantee QoS to the users. Resource availability is managed by Call Admission Control (CAC) in mobile networks.

One of the most critical problems occurs if the UE is leaving the coverage area of its serving cell and enters the new cell, which is not able to provide enough radio resources. In

This work has been supported by Grant No. P102/12/P613 funded by the Czech Science Foundation. this case, the connection is either dropped or QoS of the UE is degraded. This situation is considered by the users to be even more critical than if the connection would not be established at all [2][3]. Therefore, a part of resources at each cell must be reserved for the UEs potentially performing handover. However, the resource reservation may significantly influence overall QoS of all UEs in the network as the reserved resources cannot be used by any UE. Thus, if the amount of resources to be reserved is over-estimated, the UEs currently served by the target cell can exploit only lower amount of resources while some resources might not be utilized at all. Therefore, the amount of resources to be reserved must be carefully determined in order to avoid degradation of QoS for both currently served UEs and the UEs performing handover.

To minimize potential QoS degradation due to the reservation of resources, it is useful to predict expected amount of users performing handover and quantity of resources required by these users. The amount of users performing handover is frequently addressed in literature via mobility prediction [3][4][5]. However, the quantity of resources required by users is omitted in most cases [6] or the same amount of resources consumed at the time of prediction is expected to be spent also after the handover [7]. Similar approach is proposed in [8], where the authors assume to reserve amount of Resource Blocks (RBs) according to the type of service. However, this assumption is not valid in OFDMAbased networks, since the number of required RBs does not depend only on the required bitrate but also on the experienced SINR. Another approach is to estimate QoS/QoE (Quality of Experience) for the users [9]. Nevertheless, even for this case, the amount of RBs consumed after the handover needs to be known. So far, no prediction has been implemented or considered for standardization in $4 \mathrm{G}$ networks. One of the main reasons are accuracy of the prediction and high requirements on computation as most of the prediction approaches demand heavy computation and processing of information collected in the past. Nevertheless, with Cloud-Radio Access Networks (CRAN) like approach [10], where network management is placed in a powerful cloud, computation capabilities are no longer a limiting aspect. Also, the small cell cloud (SCC) approach [11] can be exploited for processing of prediction. Both above-mentioned approaches seem to be very promising candidates for $5 \mathrm{G}$. The implementation of advanced computing capabilities in C-RAN or SCC can be seen as a key enabler for 
exploitation of advanced procedures for mobility management based on prediction. The prediction for mobility management opens a way how to address problem of seamless connectivity, which is one of the essential technical objective in 5G [12].

In this paper, we propose a novel algorithm for prediction of SINR and CQI after handover is performed. The proposed approach exploits knowledge of system parameters related to handover and possibility to decompose the overall interference into two components. With accurate CQI prediction, the number of RBs required by the UEs after the handover can be derived and network performance can be improved.

The rest of the paper is organized as follows. Next section defines system model for the proposed prediction. Section III describes methodology for performance evaluation and presents simulation results. Last section summarizes the major findings and outlines future work.

\section{SYSTEM MODEL FOR PREDICTION}

This section describes the system model and, then, it presents a novel approach for prediction of SINR and CQI experienced by the UE after handover.

During the UE's movement, received signal strength from all cells is varying. Therefore, if the UE crosses boundary between two cells, handover to the target cell is performed. Handover in mobile network is typically initiated if the signal level observed by the UE from the serving cell $\left(s_{s}\right)$ drops below the signal level from the target cell $\left(s_{t}\right)$ at least by a hysteresis $\left(\Delta_{H M}\right)$, i.e., handover is initiated if:

$$
s_{s}<s_{t}-\Delta_{H M}
$$

Usually, this condition must hold for the time-to-trigger (TTT) interval in order to avoid ping-pong effect. For the same reason, $s_{s}$ and $s_{t}$ represent statistic values averaged over time in real networks and also in our evaluations.

In OFDMA networks, efficiency of data delivery depends not only on the level of $s_{s}$ but also on the interference caused by other neighboring cells $\left(l_{n}\right)$ and noise $(n)$. Thus, the crucial parameter for determination of the channel quality is SINR defined as:

$$
\gamma=\frac{s_{s}}{l_{n}+n}=\frac{s_{s}}{\sum_{j \neq s} s_{j}+n}
$$

where $s_{j}$ represents received signal from the $j$-th neighboring cell.

The objective of our work is to derive SINR level after the handover. To that end, we propose to decompose the overall interference after the handover $\left(l_{\mathrm{HO}}\right)$ into two parts. The first part is the interference caused by the former serving cell. The interference due to the serving cell is known very accurately as, in fact, this is a difference between $s_{t}$ and $s_{s}$, which is equal to hysteresis, i.e., $s_{t}-s_{s}=\Delta_{H M}$ (see Figure 1). The interference caused by serving cell can be influenced also by the TTT. However, this impact is negligible as the TTT value is typically in tens or hundreds of $m s$ [13]. Moreover, the TTT is indirectly proportional to the speed of users (i.e., lower value for faster user). Therefore, we can neglect the impact of the

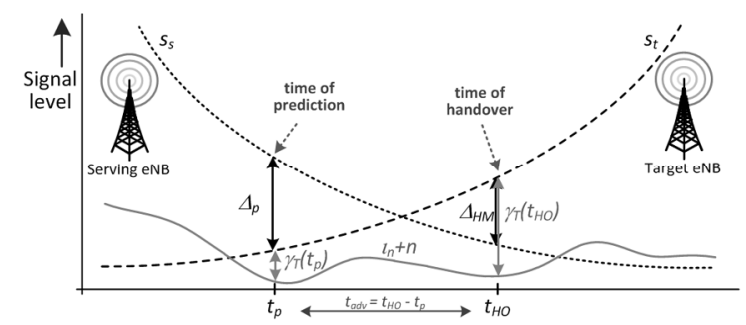

Figure 1. Principle and parameters for SINR/CQI prediction.

TTT. The second part of the $l_{H O}$ is the interference due to other (neighboring) cells and noise. This level of interference is not known and its prediction is a very complex problem. We propose to exploit knowledge of the level of interference caused by all neighboring cells but the target cell at the time when the prediction is carried out. Thus, we calculate interference of all cells except the serving and target cells $\left(l_{n}\right)$ in the time of prediction $\left(t_{p}\right)$ as:

$$
l_{n}\left(t_{p}\right)=\sum_{\substack{j \neq t \\ j \neq s}} s_{j}\left(t_{p}\right)
$$

where $s_{j}\left(t_{p}\right)$ represents the signal level measured by the UE from the $j$-th cell in the time $t_{p}$ (see Figure 1).

Knowing $l_{n}$ and quality of signal from the target cell, we define new parameter - "signal of target cell to interference plus noise ratio" $\left(\gamma_{T}\right)$ :

$$
\gamma_{T}=\frac{s_{t}}{l_{n}+n}=\frac{s_{t}}{\sum_{\substack{j \neq t \\ j \neq s}} s_{j}+n}
$$

Then, the interference at the time of handover $\left(l_{\mathrm{HO}}\right)$ is a function of the $\gamma_{T}$ and $\Delta_{H M}$. To simplify the complex problem of interference prediction, we assume the interference due to the neighboring cells is not varying significantly. This assumption holds for a short interval before the handover. The interval of validity of this assumption is investigated later in this paper.

The function describing relation between $\Delta_{H M}$ (representing impact of interference caused by the former serving cell) and $\gamma_{T}$ (representing impact of interference caused by all neighboring cells except the target one) must be found to calculate expected SINR at the handover time $\left(\gamma_{\mathrm{HO}}\right)$. In this regard, we define relative significance of both components with respect to their mutual levels. In other words, predicted $\gamma_{H O}$ depends on whether the former serving cell will be a dominant interferer with respect to the sum of interference produced by all neighboring cells or not. Three cases can be distinguished:

- First, if $\Delta_{H M} \ll \gamma_{T}$, the $\gamma_{H O}$ is more influenced by $\Delta_{H M}$ as the former serving cell will produce more significant interference than all neighboring cells together.

- Second, if $\Delta_{H M} \gg \gamma_{T}$, the $\gamma_{H O}$ should be closer to the $\gamma_{T}$ as the neighboring cells introduce higher level of interference than the former serving cell.

- Third, if $\gamma_{T} \approx \Delta_{H M}$, the $\gamma_{\text {HO }}$ will be lower than both $\Delta_{H M}$ and $\gamma_{T}$. In this situation, the final $\gamma_{H O}$ depends on mutual difference of both components. 


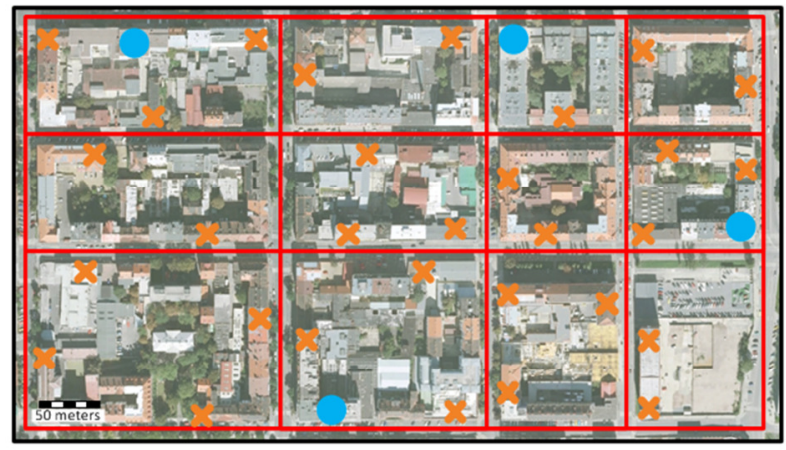

Figure 2. Simulation scenario with position of microcells (blue dots) and femtocells (orange crosses).

To reflect all three above-mentioned cases for relation between $\Delta_{H M}$ and $\gamma_{T}$, we propose the function for prediction of the $\gamma_{H O}$ as follows:

$$
\gamma_{H O}=\min \left(\Delta_{H M}, \gamma_{T}\right)-\beta \times k_{1}^{-\left|\Delta_{H M}-\gamma_{T}\right| / 10}+\mathcal{E}_{d B}
$$

where the first term, $\min \left(\Delta_{H M}, \gamma_{T}\right)$, defines upper bound of the $\gamma_{H O}$ corresponding to the lower value of both interference components; $k_{l}$ is the coefficient introducing impact of the relative difference between both interference components (we have derived the most suitable value of $k_{l}$ by simulations and, in this paper, equals to 75); and $\varepsilon_{d B}$ stands for the compensation of a prediction error in $\mathrm{dB}$ to minimize over/under-estimation of the $\gamma_{\mathrm{HO}} ; \beta$ represents the case when both former serving cell and neighboring cells cause the same level of interference $\left(\gamma_{T}=\Delta_{H M}\right)$. Parameter $\beta$ is derived as follows. Generally, sum of the interferences produced by several cells with exactly the same level of signal can be calculated as:

$$
\boldsymbol{l}_{\text {cumul }}=\boldsymbol{l}_{i}+10 \times \log _{10}\left(N_{n}\right)
$$

where $N_{n}$ represents the number of cells generating the same level of interference $\left(l_{i}\right)$. If $\gamma_{T}=\Delta_{H M}$, both sources of interference $\left(\gamma_{T}\right.$ and $\left.\Delta_{H M}\right)$ are of the same importance and $N_{n}=2$ in (6). Consequently, $\beta=10 \times \log _{10}(2)$ in

Usually, exact knowledge of $\gamma_{H O}$ is not necessary as the transmission efficiency, representing the number of bits carried per resource block $\left(b_{R B}\right)$, is expressed by modulation and coding scheme (MCS). The MCS is selected according to $\gamma_{\mathrm{HO}}$, which is indicated by CQI in LTE(-A) networks [14]. Therefore, CQI is predicted instead of exact $\gamma_{H O}$ for practical

TABLE I

SIMULATION PARAMETERS

\begin{tabular}{|l|l|}
\hline \multicolumn{1}{|c|}{ Parameter } & \multicolumn{1}{c|}{ Value } \\
\hline Carrier frequency & $2 \mathrm{GHz}$ \\
\hline Bandwidth & $20 \mathrm{MHz}$ \\
\hline Transmitting power of eNB/FAP & $27 / 15 \mathrm{dBm}$ \\
\hline Wall loss & $10 \mathrm{~dB}$ \\
\hline Path loss model from FAP/eNB & ITU-R P.1238/Okumura Hata \\
\hline Number of UEs/eNBs/FAPs & $60 / 4 /\{0-90\}$ \\
\hline Speed of UEs & $1 \mathrm{~m} / \mathrm{s}$ \\
\hline Mobility model & Manhattan-like (see [15]) \\
\hline Simulated real-time/ No. of drops & $18000 \mathrm{~s} / 5$ \\
\hline Simulation area & $640 \times 360 \mathrm{~m}$ \\
\hline
\end{tabular}

implementation. The predicted CQI $\left(C Q I_{p}\right)$ is derived as:

$$
C Q I_{p}=f\left(\gamma_{H O}\right)+\varepsilon_{C Q I}
$$

where function $f$ maps modulation and coding derived from SINR to CQI [14], and $\varepsilon_{C Q I}$ is the compensation of the prediction error. The compensation is implemented by means of manual shifting the CQI index in order to minimize over/under-estimation of the $C Q I_{p}$.

The knowledge of UE's capacity requirements $\left(c_{r e q}\right)$ and $C Q I_{p}$ enables to derive exact number of resource blocks per frame $\left(n_{R B}\right)$ to be required by the UE just after the handover:

$$
n_{R B}=\left\lceil c_{r e q} / b_{R B}\right\rceil
$$

where $\lceil\cdot\rceil$ represents ceiling function.

The $n_{R B}$ derived by our proposal is intended to be used for reservation of resources for the UE at the target cell to avoid call dropping after the handover [1][3]. In addition to this, the $n_{R B}$ can be exploited also to schedule transmission of other UEs in the network with respect to the estimated amount of consumed RBs in the future (e.g., for opportunistic delay tolerant content or for sync of content of mobile devices).

\section{PERFORMANCE EVALUATION}

Performance of the proposed prediction algorithm is evaluated by means of simulations in Matlab. In the first subsection, models, scenarios and performance metrics are defined. Simulation results are presented afterwards.

\section{A. Models, scenarios and performance metrics}

In simulations, four microcells are deployed in line with deployment of cells by Vodafone CZ in Prague, Czech Republic (see Figure 2). In addition, we randomly deploy between 0 to 90 femto access points (FAPs) inside buildings. Major simulation parameters are summarized in Tab. I.

For exploitability of predicted $\gamma_{\mathrm{HO}}$, the prediction itself must be carried out before the handover. Since exact time of handover $\left(t_{H O}\right)$ is not known in advance, we exploit definition of handover as a relative difference between $s_{s}$ and $s_{t}$, i.e., $t_{H O}$ is the time when $s_{t}-s_{s}=\Delta_{H M}$. Analogically, the time of prediction $t_{p}$ is related to the time when the difference between both signal levels drops below $\Delta_{p}=s_{s}-s_{t}$ (see Figure 1). Then, the time advance $\left(t_{a d v}\right)$ is understood as the time corresponding to the relative change between $s_{s}$ and $s_{t}$ from $\Delta_{p}$ to $\Delta_{H M}$ (see Figure 1). Therefore, the $t_{a d v}$ is proportional to $\Delta_{p}+\Delta_{H M}$. Based on our performed simulations, the average values of the $t_{a d v}$ are $1 / 5 / 13 / 23 / 35 \mathrm{~s}$ for handover to/from macrocell eNB for $\Delta_{p}$ equal to $0 / 1 / 3 / 5 / 7 \mathrm{~dB}$ and $\Delta_{H M}=0 \mathrm{~dB}$. For handover between FAPs, the $t_{a d v}$ is $1 / 4 / 9 / 15 / 22 \mathrm{~s}$ for the same values of $\Delta_{p}$ and $\Delta_{H M}$.

We average results over five simulation drops (each drop is distinguished by different random deployments of the FAPs and user's movement patterns), which last $18000 \mathrm{~s}$. We monitor SINR on frame by frame basis for MCS evaluation. The CQI is reported every $1 \mathrm{~s}$ as pedestrians, moving with speed of $1 \mathrm{~m} / \mathrm{s}$, are assumed in simulations. 
Two metrics are considered for evaluation of the proposed approach: prediction efficiency and prediction inaccuracy. The prediction efficiency, $\eta_{\text {eff, }}$ represents ratio of correct predictions. The correct prediction means that $C Q I_{p}=C Q I_{H O}$, where $C Q I_{H O}$ is the CQI really experienced by the UE just after the handover. The $\eta_{\text {eff }}$, is defined as:

$$
\eta_{\text {eff }}=n_{C Q I}^{c} /\left(n_{C Q I}^{c}+n_{C Q I}^{i}\right)
$$

where $n_{C Q I}^{c}$ and $n_{C Q I}^{i}$ represents the number of correct and incorrect predictions of CQI, respectively.

The prediction inaccuracy, $\sigma_{i a c}$, is defined as average level of inaccuracy in CQI prediction by means of error in predicted amount of bits per RB $\left(b_{R B, p}\right)$ and real amount of bits per RB assigned to the UE just after handover $\left(b_{R B, H O}\right)$ :

$$
\sigma_{i a c}=\frac{1}{\left(n_{C Q I}^{c}+n_{C Q I}^{i}\right)} \sum_{n_{C Q P}^{c}+n_{C Q P}^{i}}\left|b_{R B, p}-b_{R B, H O}\right|
$$

\section{B. Simulation results}

In this section, performance of the proposed approach for prediction of resources consumed by the UEs after the handover is assessed. The distribution of error in CQI prediction, defined as $E_{C Q I}=C Q I_{p}-C Q I_{H O}$, is presented in Figure 3. As can be seen, the probability of correct CQI prediction (i.e., $E_{C Q I}=0$ ) is influenced by prediction correction $\varepsilon_{d B}$. The negative value of $\varepsilon_{d B}$ decreases probability of correct prediction $\left(E_{C Q I}=0\right)$ and increases probability of $E_{C Q I}=-1$. This behavior is expectable as $\varepsilon_{d B}$ just shifts predicted value of $\gamma_{H O}$ (see (5)). Nevertheless, the important fact observed in this figure is that situation when $\mathrm{P}\left(E_{C Q I}=-1\right)>\mathrm{P}\left(E_{C Q I}=0\right)$ can be reached by setting appropriate $\varepsilon_{d B}$ in $\quad$ (5). It enables us to increase probability of correct prediction by joint tuning of $\varepsilon_{d B}$ and $\varepsilon_{C Q I}$ (in Figure 3, maximum efficiency is reached for $\varepsilon_{d B}=-2 d B$ and $\left.\varepsilon_{C Q I}=-1\right)$.

The further results are presented for combination of $\varepsilon_{C Q I}$ and $\varepsilon_{d B}$, which reaches the highest $\eta_{\text {eff }}$ for every density of FAPs. Deeper analysis of impact of these parameters is left for future research together with design of algorithm for dynamic adaptation of parameters according to changes in network's environment and in users' behavior.

Figure 4 shows $\eta_{\text {eff }}$ over time advance of prediction before handover $\left(\Delta_{p}\right)$ for various FAPs' densities. Subplots in Figure 4

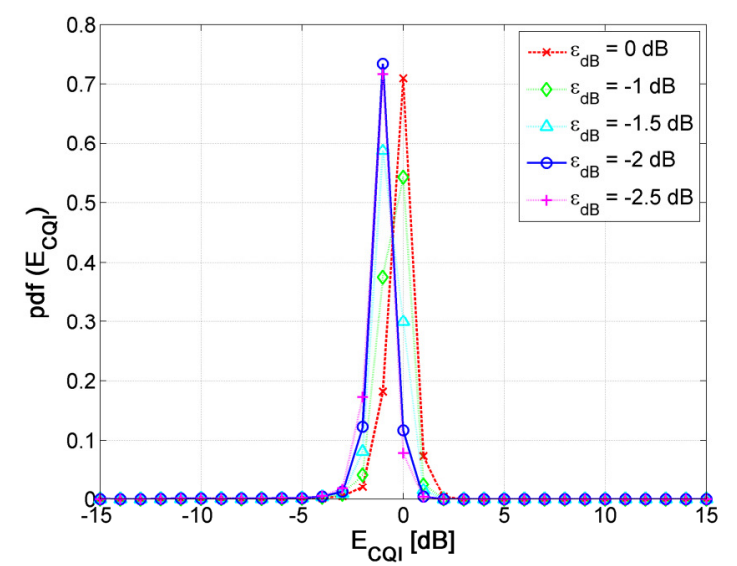

Figure 3. Probabilistic density function of $E_{C Q I}$ for $\Delta_{p}=3 d B$ and 30 FAPs.

represent cases when i) no prediction error is observed $\left(E_{C Q I}=0\right)$, ii) error is up to one CQI level $\left(\left|E_{C Q I}\right| \leq 1\right)$, and iii) error is up to two CQI levels $\left(\left|E_{C Q I}\right| \leq 2\right)$. As can be seen from Figure 4a, the ratio of correct predictions for the proposed algorithm (in figures denoted as "Prop") is decreasing with density of FAPs from roughly 95\% (no FAPs) to 93\% (90 FAPs) if $\Delta_{p}=0 d B$ and from $66 \%$ (no FAPs) to $52 \%$ (90 FAPs) if $\Delta_{p}=7 \mathrm{~dB}$. With respect to the state of art (SoA) approach described in [7] (in figures denoted as "SoA"), the $\eta_{\text {eff }}$ is improved 1.2 times for no FAPs, and 1.9 times if 90 FAPs are deployed. In the proposed approach, always more than $96 \%$ and $97.5 \%$ of predictions are within $\left|E_{C Q I}\right| \leq 1$ over all investigated range of $\Delta_{p}$ for no FAPs and 90 FAPs, respectively (see Figure $4 b$ ). Figure $4 c$ further shows that at least $97.3 \%$ (for no FAPs) and 99.7\% (90 FAPs) of predictions are within $\left|E_{C Q I}\right| \leq 2$. For competitive state of the art approach, only $81 \%$ and $94.7 \%$ predictions are within $\left|E_{C Q I}\right| \leq 1$ and $\left|E_{C Q I}\right| \leq 2$, respectively, for $\Delta_{p}=7 \mathrm{~dB}$. Therefore, we can conclude that the prediction efficiency is significantly improved by our scheme, especially, if the prediction needs to be done earlier before handover (i.e., for higher $\Delta_{p}$ ).

Figure 5 depicts the impact of density of cells and $\Delta_{p}$ on the average prediction inaccuracy, $\sigma_{i a c}$. The $\sigma_{i a c}$ increases with time advance of the prediction $\left(\Delta_{p}\right)$ but decreases with higher density of FAPs due to more homogeneous interference caused by neighbor cells. The average prediction error, expressed in

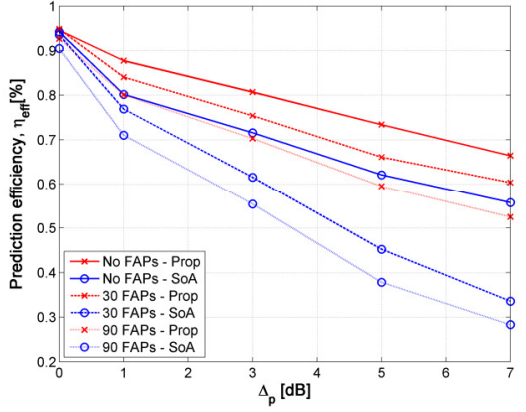

(a)

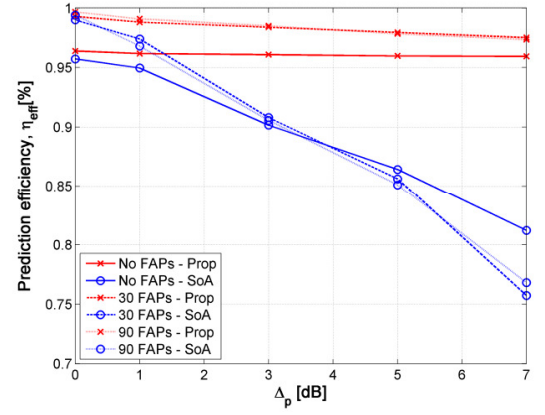

(b)

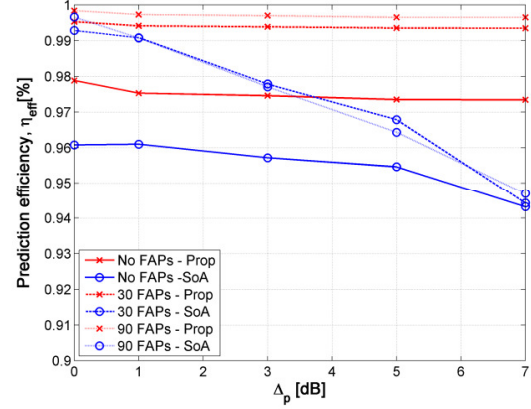

(c)

Figure 4. Prediction efficiency $\eta_{\text {eff }}$ for various acceptable error in CQI prediction ( $E_{C Q I}=0$ in (a), $\left|E_{C Q I}\right| \leq 1$ in (b), $\left|E_{C Q I}\right| \leq 2$ in (c)) 


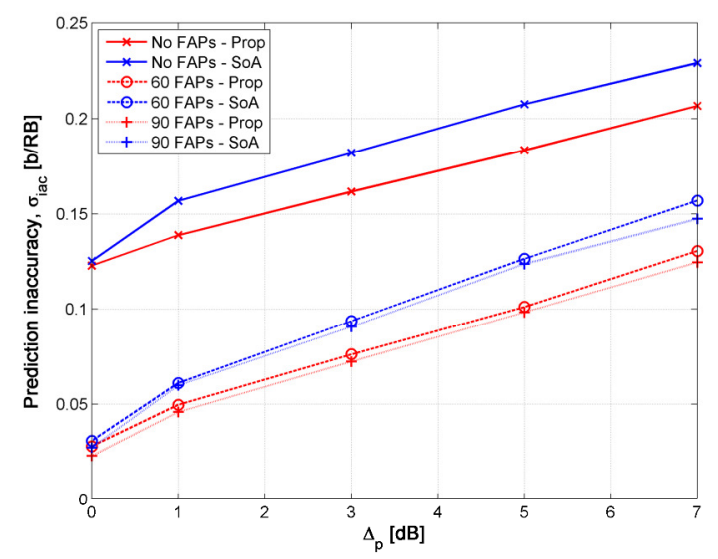

Figure 5. Impact of density of cells and $\Delta_{p}$ on prediction inaccuracy $\sigma_{\text {iac }}$.

$\mathrm{b} / \mathrm{RB}$, varies between only 0.023 and $0.21 \mathrm{~b} / \mathrm{RB}$ for our proposal while the competitive scheme reaches $\sigma_{i a c}$ from 0.027 to $0.23 \mathrm{~b} / \mathrm{RB}$. This corresponds to a gain by our proposal up to roughly $15 \%$.

\section{CONCLUSIONS AND FUTURE WORK}

The algorithm for prediction of the CQI after handover has been proposed. The prediction is based on knowledge of hysteresis defined by networks for handover and level of interference produced by neighboring cells. As the results show, the algorithm predicts CQI correctly in 52\% to $95 \%$ depending on time advance of the prediction and density of the FAPs. This efficiency is up to 1.9 times higher than in case of existing scheme and the gain increases if prediction is performed earlier before handover. Moreover, error up to one CQI level is reached in $96 \%$ for all investigated time advances and densities of the FAPs. Such precise prediction of CQI enables to exploit this approach not only for call admission control purposes in $5 \mathrm{G}$ but also for advanced scheduling and resource allocation in $5 \mathrm{G}$, especially for opportunistic nonurgent content.

In the future, we plan to enhance the proposed algorithm considering learning process for derivation of error correction parameters $\left(\varepsilon_{d B}\right.$ and $\left.\varepsilon_{C Q I}\right)$ to maximize its efficiency. Further, we plan to jointly integrate proposed channel quality prediction with handover prediction to show impact on QoS parameters such as call drops.

\section{REFERENCES}

[1] E. Calvanese Strinati et al., "Next Generation of Wireless Networks," white paper of the NetWorld 2020 ETP Expert Working Group on Next Generation of Wireless Networks, June 2014.

[2] T. Al-Khasib, L. Lampe, "Admission Control and Resource Optimization for Multiple-User OFDMA Cognitive Radio Systems," Int. Journal of Electronics and Communications, vol. 66, no. 5, May 2012.

[3] J. Martinez-Bauset, J.M. Gimenez-Guznam, V. Pla, "Optimal Admission Control in Multimedia Mobile Networks with Handover Prediction," IEEE Wireless Communications Magazine, vol. 15, no. 5, pp. 38-44, October 2008.

[4] H. Si, Y. Wang, J. Yuan and X. Shan, "Mobility Prediction in Cellular Network Using Hidden Markov Model," IEEE Consumer Communications and Networking Conference (CCNC 2010), Las Vegas, NV, January 2010.

[5] A. Noulas, S. Scellato, N. Lathia, C. Mascolo, "Mining User Mobility Features for Next Place Prediction in Location-based Services," IEEE International Conference on Data Mining (ICDM 2012), Brussels, Belgium, December 2012.

[6] L.B. Le, E. Hossain, D. Niyato, D.I. Kim, "Mobility-aware Admission Control with QoS Guarantees in OFDMA Femtocell Networks," IEEE International Conference on Communications (ICC 2013), pp. 22172222, June 2013.

[7] C. Kosta, T. Sodunke, M. Shateri, R. Tafazolli, "Two-stage call admission control policy for LTE systems," IEEE International Wireless Communications and Mobile Computing Conference (IWCMC 2010), pp. 1101-1105, 2010

[8] M. Khabazian, O. Kubbar, H. Hassanein, "Call Admission Control with Resource Reservation for Multi-service OFDM Networks," IEEE International Conference on Computing, Networking and Communications (ICNC 2012), Maui, Hawai, January 2012.

[9] T. Taleb, A. Ksentini, "QoS/QoE Predictions-based Admission Control for Femto Communications," in Proc. IEEE International Conference on Communications (ICC 2012), pp. 5146-5150, June 2012.

[10] K. Chen, R. Duan, "C-RAN: The Road Towards Green RAN," whitepaper of China Mobile Research Institute, October 2011.

[11] F. Lobillo, et al., "An architecture for mobile computation offloading on cloud-enabled LTE small cells," IEEE Wireless Communications and Networking Conference (WCNC 2014) workshops, Istanbul, Turkey, April 2014

[12] S. Chen, J. Zhao, "The requirements, challenges, and technologies for $5 \mathrm{G}$ of terrestrial mobile telecommunication," IEEE Communications Magazine, Vol. 52, No.5, May 2014.

[13] Q. Kuang1, J. Belschner, Z. Bleicher, H. Droste, J. Speidel, "A measurement-based study of handover improvement through range expansion and interference coordination," Wireless Communications and Mobile Computing, Early view, January 2014.

[14] 3GPP TS 36.213, "Physical Layer Procedures," v.12.1.0, March 2014.

[15] Z. Becvar, M. Vondra, P. Mach, "Dynamic Optimization of Neighbor Cell List for Femtocells," IEEE Vehicular Technology Conference Spring (VTC-Spring 2013), Dresden, Germany, June 2013. 